Research

Open Access

\title{
Cardiovascular response to dobutamine stress predicts outcome in severe sepsis and septic shock
}

\author{
Anand Kumar ${ }^{1,2}$, Elizabeth Schupp ${ }^{3}$, Eugene Bunnell ${ }^{3}$, Amjad Ali4 ${ }^{4}$ Barry Milcarek ${ }^{2}$ and \\ Joseph E Parrillo2
}

\begin{abstract}
${ }^{1}$ Section of Critical Care Medicine, Health Sciences Centre/St. Boniface Hospital, University of Manitoba, Winnipeg, Manitoba, Canada 2Division of Cardiovascular Disease and Critical Care Medicine, Cooper University Hospital, Robert Wood Johnson Medical School, Camden, New Jersey, USA

3Division of Cardiovascular Disease and Critical Care Medicine and Rush-Presbyterian-St. Luke's Medical Center, Chicago, Illinois, USA

${ }^{4}$ Section of Nuclear Medicine, Rush-Presbyterian-St. Luke's Medical Center, Chicago, Illinois, USA
\end{abstract}

Corresponding author: Anand Kumar, akumar61@yahoo.com

Received: 23 Apr 2007 Revisions requested: 22 Jun 2007 Revisions received: 4 Feb 2008 Accepted: 4 Mar 2008 Published: 4 Mar 2008

Critical Care 2008, 12:R35 (doi:10.1186/cc6814)

This article is online at: http://ccforum.com/content/12/2/R35

(c) 2008 Kumar et al.; licensee BioMed Central Ltd.

This is an open access article distributed under the terms of the Creative Commons Attribution License (http://creativecommons.org/licenses/by/2.0), which permits unrestricted use, distribution, and reproduction in any medium, provided the original work is properly cited.

\begin{abstract}
Introduction During septic shock, resistance to the haemodynamic effects of catecholamine vasopressors and inotropes is a well-recognised marker of mortality risk. However, the specific cardiovascular or metabolic response elements that are most closely associated with outcome have not been well defined. The objective of this study was to assess cardiovascular and metabolic responses to dobutamine as correlates of outcome in patients with severe sepsis or septic shock.

Methods A prospective, non-randomised, non-blinded interventional study of graded dobutamine challenge $(0,5,10$, and $15 \mu \mathrm{g} / \mathrm{kg} / \mathrm{min}$ ) in adult patients who had undergone pulmonary artery catheterisation within 48 hours of onset of severe sepsis or septic shock (8 survivors/15 non-survivors) was performed. Radionuclide cineangiography during graded infusion was used to determine biventricular ejection fractions at each increment of dobutamine.
\end{abstract}

Results In univariate analysis, a variety of cardiovascular or haemodynamic and oxygen transport or metabolic variables (at the point of maximum cardiac index response for a given subject) were associated with survival including: increased stroke volume index $(p=0.0003)$; right ventricular end-diastolic volume index $(p=0.0047)$; left ventricular stroke work index ( $p$ $=0.0054)$; oxygen delivery index $(p=0.0084)$; cardiac index $(p$ $=0.0093$ ); systolic blood pressure/left ventricular end-systolic volume index ratio $(p=0.0188)$; left ventricular ejection fraction $(p=0.0160)$; venous oxygen content $(p=0.0208)$; mixed venous oxygen saturation $(p=0.0234)$; pulse pressure $(p=$ $0.0403)$; decreased pulmonary artery diastolic pressure $(p=$ $0.0133)$; systemic vascular resistance index $(p=0.0154)$; extraction ratio $(p=0.0160)$; and pulmonary vascular resistance index $(p=0.0390)$. Increases of stroke volume index of greater than or less than $8.5 \mathrm{~mL} / \mathrm{m}^{2}$ were concordant with survival or death in 21 of 23 cases. Multivariate profile construction showed stroke volume index as the dominant discriminating variable for survival with the systolic blood pressure/left ventricular end-systolic volume index ratio alone among all other variables significantly improving the model.

Conclusion Survivors maintain cardiac responsiveness to catecholamine stimulation during septic shock. Survival from severe sepsis or septic shock is associated with increased cardiac performance and contractility indices during dobutamine infusion. Further studies are required to determine whether these parameters are predictive of outcome in a larger severe sepsis/septic shock population.

\footnotetext{
$\mathrm{Cl}=$ cardiac index; $\mathrm{CO}=$ cardiac output $\mathrm{CvO} 2=$ venous oxygen content $\mathrm{CVP}=$ central venous pressure; $\mathrm{DO} 2 \mathrm{I}=$ oxygen delivery index; $\mathrm{dPAP}=$ diastolic pulmonary artery pressure; $E C G=$ electrocardiographic; $E D V=$ end-diastolic volume; $E F=$ ejection fraction; $E S V=$ end systolic volume; $\mathrm{FiO}_{2}=$ inspired oxygen fraction; $\mathrm{HR}=$ heart rate; LVEDVI/PWP = left ventricular end-diastolic volume index: pulmonary wedge pressure ratio; LVEF $=$ left ventricular ejection fraction; $\mathrm{LVSWI}=$ left ventricular stroke work index; $\mathrm{MAP}=$ mean arterial pressure; $\mathrm{mPAP}=$ mean pulmonary artery pressure; $\mathrm{MVO} 2=$ mixed venous oxygen saturation; $\mathrm{OER}=$ oxygen extraction ratio; $\mathrm{PaO}_{2}=$ arterial partial pressure of oxygen; $\mathrm{PAP}=$ pulmonary artery pressure; $\mathrm{pCO}_{2}=$ partial pressure of carbon dioxide; $\mathrm{pO}_{2}=$ partial pressure of oxygen; $\mathrm{PP}=$ pulse pressure; $\mathrm{PWP}=$ pulmonary wedge pressure; $\mathrm{PVR}=$ pulmonary vascular resistance; PVRI = pulmonary vascular resistance index; RAP = right arterial pressure; RVEDV = right ventricular end-diastolic volume; RVEDVI/RAP = right ventricular end-diastolic volume: right atrial pressure ratio; RVEF = right ventricular ejection fraction; RVSWI = right ventricular stroke work index; sBP/LVESVI = peak systolic pressure: left ventricular end-systolic volume index ratio; sPAP/RVESVI = peak systolic pulmonary artery pressure: $\mathrm{SV}=$ stroke volume; $\mathrm{SVI}=$ stroke volume index; $\mathrm{SVR}=$ systemic vascular resistance; $\mathrm{SVR}=$ systemic vascular resistance index; $\mathrm{VO}_{2} \mathrm{I}=$ oxygen consumption index; right ventricular end-systolic volume index ratio.
} 


\section{Introduction}

The cardiovascular profile of septic shock has been well defined over the last 30 years. One of the major areas of investigation has been the cardiovascular correlates of outcome. Early clinical studies, prior to the widespread adoption of the balloon-tipped pulmonary artery flotation catheter with thermodilution capability in the 1980 s, tended to suggest that fatal sepsis and septic shock were characterised by a low cardiac output (CO) and high systemic vascular resistance (SVR) [1]. However, the concept of septic shock as a hyperdynamic circulatory state characterised by high cardiac output and low systemic vascular resistance eventually became well established $[2,3]$. Several baseline cardiovascular variables have been proposed to differentiate between survivors and non-survivors of septic shock including heart rate (HR), CO, SVR and oxygen delivery index $\left(\mathrm{DO}_{2} \mathrm{l}\right)$ [4-7]. Studies using radionuclide cineangiography and volumetric echocardiography have further demonstrated an association between survival and biventricular dilatation and ejection fraction (EF) depression [3,810]. Evidence has also accumulated suggesting that fatal septic shock is associated with decreased responsiveness to fluid resuscitation $[11,12]$.

Bedside experience and several clinical studies suggest that blunted cardiovascular responsiveness to catecholamine support is also a marker of high risk of death in sepsis and septic shock [13]. However, the relationship of ventricular ejection fraction and/or ventricular volume responses and other ventricular volume-related cardiovascular variables under dobutamine stress to survival in sepsis and septic shock is undefined. This study was designed to assess ventricular and cardiovascular performance, oxygen transport, and oxygen delivery responses during dobutamine challenge and their association with survival in sepsis.

\section{Materials and methods}

This study received Institutional Review Board approval. Informed consent was obtained from all subjects or appropriate representatives prior to enrolment.

Adult (more than 18 years of age) Intensive Care Unit patients were recruited into this prospective, non-randomised, open interventional study. In order to be eligible, patients were required to have developed severe sepsis or septic shock within the preceding 48 hours and to have both a pulmonary artery catheter and arterial pressure catheter in place (based on clinical requirements as defined by the attending physician). Study eligibility was further contingent on confirmation of correct placement of the pulmonary artery catheter based on appropriate pressure traces on insertion and position on chest radiography.
The presence of severe sepsis was defined by 1991 SCCM/ ACCP Consensus Statement on Sepsis Definitions [14]. These criteria included: the presence of suspected or documented infection; three of the following four systemic inflammatory response elements temperature $>38^{\circ} \mathrm{C}$ or $<36^{\circ} \mathrm{C}$, heart rate $>90$ beats $/ \mathrm{min}$, respiratory rate $>20$ breaths $/ \mathrm{min}$ or $\mathrm{PaCO}_{2}<32 \mathrm{~mm} \mathrm{Hg}$ and white blood cell count $>12,000$ cells/ $\mathrm{mm}^{3},<4,000$ cells $/ \mathrm{mm}^{3}$ or $>10$ per cent immature (band) forms; and evidence of at least one organ failure.

Septic shock was defined by the presence of post-fluid resuscitation hypotension (mean arterial pressure $<65 \mathrm{mmHg}$ ) necessitating vasopressor support.

Patients with known ischemic cardiac disease and mixed shock states with clinically significant primary cardiogenic, haemorrhagic or pulmonary embolic elements were excluded.

After consent was obtained, each patient underwent graded dobutamine challenge as follows: If required, subjects initially received saline infusion to a pulmonary artery wedge pressure (PWP) of $15 \mathrm{mmHg}$ as required because pilot studies had shown the potential for significant hypotension with dobutamine absent adequate intravascular volume as evidenced by PWP of 15 or higher. After documentation of a PWP of 15 $\mathrm{mmHg}$ or higher, baseline haemodynamic and vital parameters (on clinically indicated doses of vasopressors) were obtained. These included heart rate (HR), systemic arterial pressures, central venous pressure (CVP), pulmonary artery pressure (PAP), pulmonary wedge pressure (PWP) and CO. Thermodilution cardiac outputs were measured by the averaging of three of four successive injections of $10 \mathrm{~mL}$ of cold (6 to $10^{\circ} \mathrm{C}$ ) dextrose 5 per cent in water at end-expiration (with the outlying values discarded). Recorded values for PAP, PWP and right atrial pressure (RAP) were also obtained at end-expiration from graphic recordings. Right and left ventricular ejection fractions (EFs) were assessed through first-pass gated technetium ${ }^{99}$ cineangiography as described below. In addition, simultaneous arterial and mixed venous blood samples (distal pulmonary artery port) were obtained for analysis of the serum $\mathrm{pH}$, partial pressure of oxygen $\left(\mathrm{pO}_{2}\right)$, partial pressure of carbon dioxide $\left(\mathrm{pCO}_{2}\right)$, and oxygen saturation (Bayer-CIBA Model 865, Tarrytown, NY).

After baseline values were obtained, a graded dobutamine challenge was performed. Dobutamine infusion through the pulmonary artery catheter introducer port was initiated at $5 \mu \mathrm{g} /$ $\mathrm{kg} / \mathrm{min}$. After 15 minutes, repeat non-invasive and invasive haemodynamic parameters were obtained over approximately five minutes. At this time, subjects also underwent repeat first pass radionuclide assessment of biventricular ejection fractions and arterial and mixed venous blood gas analysis. The dobutamine infusion rate was increased by $5 \mu \mathrm{g} / \mathrm{kg} / \mathrm{min}$ at 20 
minute intervals to a maximum of $15 \mu \mathrm{g} / \mathrm{kg} / \mathrm{min}$ with the assessment process repeated during the final five minutes of each interval. During the infusion, fluid and additional vasopressor support (beyond baseline needs) to maintain a minimum PWP of $15 \mathrm{mmHg}$ and a mean arterial pressure (MAP) of $65 \mathrm{mmHg}$ was mandated; however, no subject required such support. During the period of the study environmental stimulation was minimised. All subjects were continuously monitored with continuous electrocardiographic (ECG) monitoring and pulse oximetry according to standard protocol in the intensive care unit. The protocol required discontinuation of dobutamine if any transient adverse effects were noted including significantly increased ventricular ectopy or decreased blood pressure. After study completion, dobutamine was tapered over 20 minutes and then discontinued. The patient's subsequent intensive care and hospital course were directed by normal clinical practice.

\section{Radionuclide Cineangiography}

Sequential measurement of biventricular ejection fraction was performed by repeat first-pass radionuclide cineangiography using technetium ${ }^{99}$-DPTA. Tc ${ }^{99}$-DPTA was injected as a tight bolus into the central veins using the pulmonary artery catheter introducer. In this study, the baseline radionuclide tracer dose was $2 \mathrm{mCi}$ with subsequent increments to 5,12 and $25 \mathrm{mCi}$. The study was performed in a $30^{\circ}$ right anterior oblique projection with a slant hole collimator fitted on to a small field gamma camera interfaced with a dedicated computer system (ICON, Siemens, Gammasonic). The data was acquired in frame mode with 440 frames, each of 60 milliseconds duration. The first transit cardiac data was reformatted into a multi-gated study using the subject's electrocardiogram recorded with the first pass data. This method provides independent cinematic display of the right as well as left ventricle. Ejection fractions (EFs) are calculated from the reformatted gated first pass studies using standard dual region of interest and background correction $[15,16]$.

Stroke volume (SV) was derived by dividing thermodilution $\mathrm{CO}$ by the concomitant HR. End-diastolic volume (EDV) was obtained by dividing SV by EF and end-systolic volume (ESV) was calculated as EDV-SV. SVR was calculated as 79.9(MAP-RAP)/CO and pulmonary vascular resistance (PVR) as 79.9(mPAP-PWP)/CO where MAP = mean arterial pressure and mPAP $=$ mean pulmonary artery pressure. Where appropriate, cardiovascular variables were indexed to body surface area.

\section{Data analysis}

Haemodynamic and oxygen transport/consumption variables at the baseline and at each dobutamine infusion rate were pooled for all subjects to derive means and standard errors of the mean. The infusion rate at the point of maximum cardiac index $(\mathrm{Cl})$ during dobutamine administration was determined for each subject and the corresponding haemodynamic/clini- cal/oxygen metabolism values at that point were used for comparison to baseline (before dobutamine but after fluid resuscitation) values. Haemodynamic values at the point of maximum $\mathrm{Cl}$ response were compared to pre-dobutamine infusion baseline values as correlates of survival to hospital discharge using two-tailed paired Student's t-test analysis. Fisher's Exact test was used for proportional analysis and independent-samples t-tests with Levene correction for unequal group variances was used for continuous measures (Tables 1 and 2). Stepwise discriminant function methods were used to assess the independent impact of 18 haemodynamic and oxygen metabolism variables identified by univariate significance test results $(p<0.2)$ as potentially associated with survival outcome. Variables defined by discriminant analysis were entered as predictors in a multiple logistic regression model with outcome as the dependent variable (Table 3). Statistical analysis was done with SPSS version 15.0.1. All significance tests are two-tailed. Statistical significance was defined as $p<0.05$. Aggregate data is expressed as mean \pm standard deviation.

\section{Results \\ Subject characteristics}

Twenty-three subjects (10 male, 13 female) were recruited for the study. Average ( \pm standard error) age was $62.6 \pm 4.0$ years. Only eight of twenty-three (35 per cent) patients with severe sepsis survived to hospital discharge. Median and inter-quartile survival duration from the day of the study among non-survivors was six days and two to nine days respectively. No clinically significant adverse effects occurred during the course of the experimental dobutamine challenge. Similar proportions of subjects in each group failed to tolerate the highest planned infusion rate $(15 \mu \mathrm{g} / \mathrm{kg} / \mathrm{min}$ ) of dobutamine (two of eight survivors and five of fourteen non-survivors). In all but one case (a non-survivor), the next highest dose $(10 \mu \mathrm{g} / \mathrm{kg} / \mathrm{min}$ ) was tolerated.

Subject characteristics of the entire group, survivors and nonsurvivors are shown in Table 1. Survivors and non-survivors were comparable in terms of average age and gender breakdown. Twenty of twenty-three subjects required pressors at the time of entry into the study. Both the proportion requiring pressor support and the degree of pressor requirement was similar in both groups. Inspired oxygen requirement was also similar in both groups. There were no significant differences in the two groups with respect to the arterial partial pressure of oxygen/inspired oxygen fraction $\left(\mathrm{PaO}_{2} / \mathrm{FiO}_{2}\right)$ ratio. The presence of renal and other individual organ failures was similar between the groups but the mean number of organ failures at the time of study entry was significantly higher in non-survivors. Similarly, the Simplified Acute Physiology Score II (SAPS II) at the time of the study were significantly higher in non-survivors; Acute Physiology and Chronic Health Evaluation II (APACHE II) scores were not. The most common underlying diagnoses in non-survivors were a post-operative state and malignancy. 
Table 1

Baseline Clinical Characteristics and Laboratory Values of Subjects

\begin{tabular}{|c|c|c|c|c|}
\hline & All $(n=23)$ & Non-survivors $(n=15)$ & Survivors $(n=8)$ & $P$ value \\
\hline Male/female & $11 \mathrm{M} ; 12 \mathrm{~F}$ & $7 \mathrm{M} ; 8 \mathrm{~F}$ & $4 \mathrm{M} ; 4 \mathrm{~F}$ & NS \\
\hline Age & $62.6 \pm 4.0$ & $61.4+5.5$ & $64.8 \pm 5.6$ & NS \\
\hline Mechanical ventilation & $17 / 23$ & $13 / 15$ & $4 / 8$ & NS \\
\hline $\mathrm{PO}_{2} / \mathrm{FiO}_{2}$ & $171.8 \pm 20.8$ & $147.2 \pm 21.1$ & $217.9 \pm 42.4$ & NS \\
\hline Pressor use & $20 / 23$ & $13 / 15$ & $7 / 8$ & NS \\
\hline Dopamine dose ( $\mu \mathrm{g} / \mathrm{kg} / \mathrm{min})$ & $6.8 \pm 1.6$ & $7.6 \pm 2.5$ & $5.9+1.8$ & NS \\
\hline Norepinephrine dose $(\mu \mathrm{g} / \mathrm{kg} / \mathrm{min})$ & $0.22 \pm 0.05$ & $0.17 \pm 0.05$ & $0.30 \pm 0.10$ & NS \\
\hline Number of organ failures & $5.3 \pm 0.3$ & $5.9 \pm 0.3$ & $4.3 \pm 0.5$ & 0.0138 \\
\hline APACHE II & $27.3 \pm 1.5$ & $28.9 \pm 1.8$ & $24.4 \pm 2.6$ & NS \\
\hline SAPS & $55.2 \pm 2.8$ & $61.5 \pm 11.3$ & $43.4 \pm 2.7$ & 0.0002 \\
\hline Serum creatinine $(\mathrm{mg} / \mathrm{dL})$ & $2.4 \pm 0.3$ & $2.4 \pm 0.4$ & $2.4 \pm 0.5$ & NS \\
\hline Serum albumin (g/dL) & $2.3 \pm 0.1$ & $2.2 \pm 0.1$ & $2.7 \pm 0.2$ & 0.0498 \\
\hline Serum bilirubin $(\mathrm{mg} / \mathrm{dL})$ & $3.2 \pm 1.2$ & $4.5 \pm 1.7$ & $0.7 \pm 0.1$ & 0.0444 \\
\hline Platelets $\left(\times 10^{3}\right.$ per uL) & $171 \pm 32$ & $119 \pm 31$ & $269 \pm 60$ & 0.0483 \\
\hline PT & $15.9 \pm 0.7$ & $17.1 \pm 0.9$ & $13.8 \pm 0.7$ & 0.0069 \\
\hline Serum lactate $(\mathrm{mmol} / \mathrm{L})$ & $2.7 \pm 0.3$ & $2.9 \pm 0.5$ & $2.5 \pm 0.5$ & NS \\
\hline Bacteraemia & $10 / 23$ & $7 / 15$ & $3 / 8$ & NS \\
\hline Organisms & $\begin{array}{l}4 \text { S. aureus } \\
3 \text { E.coli } \\
1 \text { S. pneumoniae } \\
1 \text { P. mirabilis } \\
1 \text { C. albicans }\end{array}$ & $\begin{array}{l}4 \text { S. aureus } \\
1 \text { E. coli } \\
1 \text { P. mirabilis } \\
1 \text { C. albicans }\end{array}$ & $\begin{array}{l}2 \text { E. coli } \\
1 \mathrm{~S} . \text { pneumoniae }\end{array}$ & \\
\hline Underlying disorder & $\begin{array}{c}5 \text { post-op } \\
4 \text { cancer } \\
2 \text { diabetes } \\
1 \text { each pancreatitis, sickle cell crisis, AIDS, } \\
\text { CVA, trauma, cirrhosis, dementia, epilepsy } \\
4 \text { nil }\end{array}$ & $\begin{array}{c}4 \text { post-op } \\
4 \text { cancer } \\
1 \text { each pancreatitis, sickle } \\
\text { cell crisis, AIDS, CVA, } \\
\text { trauma, cirrhosis, nil }\end{array}$ & $\begin{array}{c}3 \text { nil } \\
2 \text { diabetes } \\
1 \text { each post-op, } \\
\text { dementia, epilepsy }\end{array}$ & \\
\hline Clinical infection & $\begin{array}{c}13 \text { pneu } \\
5 \text { bsi } \\
2 \text { uti } \\
2 \text { peritonitis } \\
1 \text { ssti }\end{array}$ & $\begin{array}{c}7 \text { pneu } \\
5 \text { bsi } \\
1 \text { uti, ssti, peritonitis }\end{array}$ & $\begin{array}{c}6 \text { pneu } \\
1 \text { uti } \\
1 \text { peritonitis }\end{array}$ & \\
\hline
\end{tabular}

Among survivors, an absence of a major primary underlying disease condition was most common. Pneumonia was the most common underlying infection in both groups accounting for seven of fifteen and six of eight cases in non-survivors and survivors respectively.

\section{Cardiovascular and haemodynamic variables}

Mean difference tests for baseline and dobutamine-stimulated haemodynamic/oxygen transport variables in survivors and non-survivors are shown in Table 2. Among baseline cardiovascular/haemodynamic or oxygen transport variables, only HR was associated with outcome $(p=0.0259)$. Higher values were associated with increased mortality risk. A cut-off value of 95 beats/min was associated with the optimal correlation with eight mismatches, three survivors and five non-survivors (Table 2, Figure 1a).

Survivors and non-survivors exhibited divergent responses to dobutamine in several different areas (Table 2, Figure 2a, 3a, $4 \mathrm{a}, 5 \mathrm{a})$. These areas can broadly be categorised as: 1) cardiac performance including cardiac index $(\mathrm{Cl})$ and stroke volume index (SVI); 2) oxygen consumption/delivery including mixed venous oxygen saturation $\left(\mathrm{MVO}_{2}\right)$, venous oxygen content $\left(\mathrm{CvO}_{2}\right)$, oxygen delivery index $\left(\mathrm{DO}_{2} \mathrm{l}\right)$ and oxygen extraction ratio (OER); 3) peripheral resistance including diastolic pulmonary artery pressure (dPAP), systemic vascular resistance index (SVRI) and pulmonary vascular resistance index (PVRI); 4) left ventricular contractility including left ventricular ejection 


\begin{tabular}{|c|c|c|c|c|c|c|}
\hline & $\begin{array}{c}\text { Survivor } \\
\text { Baseline } \\
(n=8)\end{array}$ & $\begin{array}{c}\text { Non-survivors } \\
\text { Baseline } \\
(n=15)\end{array}$ & $\begin{array}{l}\text { P value: survivor } \\
\text { vs non-survivor- } \\
\text { baseline }\end{array}$ & $\begin{array}{l}\text { Survivor: } \\
\text { change from } \\
\text { baseline }\end{array}$ & $\begin{array}{l}\text { Non-survivor: } \\
\text { change from } \\
\text { baseline }\end{array}$ & $\begin{array}{l}\mathrm{P} \text { value: survivor vs } \\
\text { non-survivor-change } \\
\text { from baseline }\end{array}$ \\
\hline $\mathrm{HR}(\min -1)$ & $88.3 \pm 5.1$ & $106.7 \pm 5.7$ & .0254 & $16.1 \pm 4.7$ & $14.9 \pm 4.2$ & .8645 \\
\hline $\mathrm{sBP}(\mathrm{mmHg})$ & $124.3 \pm 6.8$ & $114.0 \pm 4.9$ & .2427 & $24.6 \pm 12.6$ & $8.3 \pm 5.2$ & .1630 \\
\hline $\mathrm{dBP}(\mathrm{mmHg})$ & $64.1 \pm 4.2$ & $57.6 \pm 2.8$ & .2207 & $-1.6 \pm 3.2$ & $2.3 \pm 2.4$ & .3508 \\
\hline $\mathrm{PP}(\mathrm{mmHg})$ & $60.1 \pm 6.5$ & $56.4 \pm 4.7$ & .6541 & $26.3 \pm 10.4$ & $5.9 \pm 4.1$ & .0403 \\
\hline $\mathrm{MP}(\mathrm{mmHg})$ & $84.2 \pm 4.2$ & $76.4 \pm 2.8$ & .1391 & $7.1 \pm 6.0$ & $4.3 \pm 3.0$ & .6225 \\
\hline SVI (ml/beat/m²) & $31.5 \pm 3.0$ & $29.0 \pm 3.0$ & .5663 & $16.4 \pm 3.7$ & $3.0 \pm 1.0$ & .0003 \\
\hline $\mathrm{Cl}\left(\mathrm{L} / \mathrm{min} / \mathrm{m}^{2}\right)$ & $2.78 \pm 0.32$ & $3.01 \pm 0.33$ & .5910 & $2.24 \pm 0.55$ & $0.80 \pm 0.22$ & .0093 \\
\hline $\mathrm{RAP}(\mathrm{mmHg})$ & $13.6 \pm 1.9$ & $14.5 \pm 1.4$ & .7346 & $-0.8 \pm 0.9$ & $0.4 \pm 0.8$ & .9405 \\
\hline sPAP $(\mathrm{mmHg})$ & $44.6 \pm 4.6$ & $40.5 \pm 2.2$ & .4459 & $3.5 \pm 5.1$ & $2.2 \pm 2.1$ & .6389 \\
\hline $\mathrm{dPAP}(\mathrm{mmHg})$ & $22.6 \pm 2.9$ & $19.5 \pm 0.8$ & .3253 & $-5.1 \pm 1.8$ & $0.6 \pm 1.1$ & .0133 \\
\hline PAM (mmHg) & $30.0 \pm 3.3$ & $26.5 \pm 0.9$ & .3494 & $-2.3 \pm 2.5$ & $1.1 \pm 1.2$ & .2442 \\
\hline PWP (mmHg) & $19.8 \pm 2.8$ & $17.6 \pm 1.1$ & .4896 & $-1.8 \pm 1.4$ & $-1.1 \pm 0.8$ & .5829 \\
\hline $\mathrm{PAO}_{2}(\mathrm{mmHg})$ & $95.6 \pm 12.2$ & $97.5 \pm 9.4$ & .9034 & $-2.3 \pm 8.2$ & $-12.2 \pm 9.2$ & .4518 \\
\hline $\mathrm{PACO}_{2}(\mathrm{mmHg})$ & $35.6 \pm 1.5$ & $32.0 \pm 2.3$ & .2000 & $-1.4 \pm 0.9$ & $-0.3 \pm 0.8$ & .3846 \\
\hline $\mathrm{O}_{2}$ sat $(\%)$ & $95.2 \pm 1.2$ & $96.0 \pm 0.7$ & .4750 & $-0.6 \pm 1.2$ & $-1.0 \pm 0.7$ & .6463 \\
\hline $\mathrm{MVO}_{2}(\%$ & $62.3 \pm 3.7$ & $62.1 \pm 2.8$ & .9879 & $8.8 \pm 2.0$ & $2.0 \pm 1.6$ & .0234 \\
\hline LVEF (\%) & $50 \pm 5$ & $57 \pm 4$ & .3184 & $11.8 \pm 3.8$ & $1 \pm 2$ & .0160 \\
\hline LVEDVI $\left(m \mathrm{Lm}^{2}\right)$ & $65.2 \pm 6.5$ & $52.7 \pm 5.8$ & .1691 & $19.9 \pm 7.4$ & $7.8 \pm 5.4$ & .1987 \\
\hline LVESVI (mL/m²) & $33.6 \pm 6.7$ & $23.0 \pm 4.4$ & .2535 & $3.5 \pm 6.5$ & $5.1 \pm 5.0$ & .8497 \\
\hline LVSWI (g.m/m²) & $28.3 \pm 4.1$ & $22.9 \pm 2.3$ & .2567 & $22.3 \pm 7.1$ & $5.2 \pm 1.4$ & .0054 \\
\hline SVRI (dynes/sec/cm²/m²) & $2232 \pm 314$ & $1835 \pm 170$ & .2728 & $-901 \pm 246$ & $-162 \pm 150$ & .0154 \\
\hline RVEF (\%) & $65 \pm 3$ & $64 \pm 4$ & .8489 & $4.8 \pm 4.1$ & $2.9 \pm 2.5$ & .6793 \\
\hline RVEDVI $\left(\mathrm{mL} / \mathrm{m}^{2}\right)$ & $48.4 \pm 4.2$ & $46.1 \pm 4.7$ & .7194 & $25.2 \pm 8.8$ & $1.7 \pm 2.6$ & .0047 \\
\hline RVESVI (mL/m²) & $16.8 \pm 2.6$ & $17.4 \pm 3.0$ & .8874 & $8.7 \pm 6.7$ & $-1.0 \pm 2.4$ & .1125 \\
\hline RVSWI (g.m/m²) & $6.8 \pm 1.1$ & $4.5 \pm 0.6$ & .0916 & $1.8 \pm 0.7$ & $1.4 \pm 0.6$ & .4365 \\
\hline PVRI (dynes/sec/cm²/m²) & $322 \pm 50$ & $263 \pm 45$ & .3910 & $-147 \pm 39$ & $-21.0 \pm 32$ & .0390 \\
\hline $\mathrm{CaO}_{2}(\mathrm{~mL} / \mathrm{dL})$ & $13.4 \pm 1.0$ & $12.8 \pm 0.5$ & .6190 & $-0.1 \pm 0.2$ & $-0.1 \pm 0.1$ & .8551 \\
\hline $\mathrm{CvO}_{2}(\mathrm{~mL} / \mathrm{dL})$ & $8.6 \pm 0.6$ & $8.4 \pm 0.6$ & .8161 & $1.3 \pm 0.4$ & $0.3 \pm 0.2$ & .0208 \\
\hline $\mathrm{DO}_{2} \mathrm{I}\left(\mathrm{mL} / \mathrm{min} / \mathrm{m}^{2}\right)$ & $352 \pm 24$ & $383 \pm 45$ & .5448 & $301 \pm 78$ & $98 \pm 29$ & .0084 \\
\hline $\mathrm{VO}_{2} \mathrm{I}\left(\mathrm{mL} / \mathrm{min} / \mathrm{m}^{2}\right)$ & $122 \pm 10$ & $122 \pm 6$ & .9967 & $32 \pm 10$ & $22 \pm 7$ & .3652 \\
\hline sbp/lvesvi (mm Hg/mL/m²) & $4.57 \pm 0.73$ & $7.33 \pm 1.51$ & .1152 & $3.12 \pm 1.32$ & $0.15 \pm 0.5$ & .0188 \\
\hline PAS/rvesvi (mm Hg/mL/m²) & $3.06 \pm 0.44$ & $3.21 \pm 0.51$ & .8255 & $0.69 \pm 0.69$ & $0.71 \pm 0.90$ & .9877 \\
\hline Ivedvi/pwp (mL/m²/mmHg) & $3.82 \pm 0.67$ & $3.18 \pm 0.38$ & .4123 & $1.52 \pm 0.47$ & $0.74 \pm 0.35$ & .1964 \\
\hline RVEDVI/RAP $\left(\mathrm{mL} / \mathrm{m}^{2} / \mathrm{mmHg}\right)$ & $4.24 \pm 0.89$ & $3.44 \pm 0.40$ & .4288 & $3.63 \pm 2.34$ & $0.23 \pm 0.46$ & .0783 \\
\hline $\mathrm{pH}$ & $7.38 \pm 0.3$ & $7.41 \pm 0.02$ & .3988 & $0 \pm 0.01$ & $0 \pm 0.01$ & .8820 \\
\hline Qs/QT & $0.11 \pm 0.02$ & $0.11 \pm 0.02$ & .8990 & $0.07 \pm 0.05$ & $0.03 \pm 0.02$ & .4793 \\
\hline OER & $0.35 \pm 0.03$ & $0.35 \pm 0.03$ & .9928 & $-0.1 \pm 0.03$ & $-0.03 \pm 0.01$ & .0160 \\
\hline
\end{tabular}


Table 3

Multiple logistic regression results*

\begin{tabular}{|c|c|c|c|c|c|c|}
\hline & \multirow[t]{2}{*}{$\mathrm{B}$} & \multirow[t]{2}{*}{ SE } & \multirow[t]{2}{*}{ Sig } & \multirow[t]{2}{*}{$\operatorname{Exp}(B)$} & \multicolumn{2}{|c|}{$85 \% \mathrm{Cl}$} \\
\hline & & & & & Lower & Upper \\
\hline$\Delta \mathrm{SVI}$ & 0.54 & 0.27 & 0.05 & 1.72 & 1.32 & 2.12 \\
\hline$\Delta s B P / L V E S V I$ & 0.45 & 0.36 & 0.19 & 1.57 & 1.03 & 2.1 \\
\hline Constant & -5.13 & 2.24 & 0.02 & 0.01 & & \\
\hline
\end{tabular}

Overall \% Correctly Classified: $95.7 \%(n=22 / 23)$

${ }^{*} \Delta \mathrm{SVI}$ and $\triangle \mathrm{SBP} / \mathrm{LVESVI}$ identified as unique predictors by discriminant function analysis Structure coefficients: $\Delta S V I=0.81, \Delta s B P / L V E S V I=$ 0.46

$\Delta$ SVI and $\triangle$ SBP/LVESVI simultaneous entry with $p \leq 0.15$ for inclusion and $p \leq 0.20$ for model retention. Overall $p=0.00004, X^{2}=20.16681, d f$

$=2$

$\Delta=$ change

fraction (LVEF), peak systolic pressure/left ventricular endsystolic volume index ratio (sBP/LVESVI) and left ventricular stroke work index (LVSWI); and 5) right ventricular lusitropy including right ventricular end-diastolic volume (RVEDVI) and right ventricular end-diastolic volume:right atrial pressure ratio (RVEDVI/RAP).

Although baseline heart rate was associated with outcome, heart rate response to dobutamine was not (Table 2, Figure $2 b)$. Both survivors and non-survivors had similar absolute and relative increases in heart rate with dobutamine stimulation. Stroke volume index response had the strongest relationship to outcome with survivors generating more than five-fold more than the approximate mean $3 \mathrm{~mL}$ increase generated by nonsurvivors (Table 2). Interestingly, only two patients demonstrated a decrease in SVI (and $\mathrm{Cl}$ ) with dobutamine infusion.

\section{Figure 1}
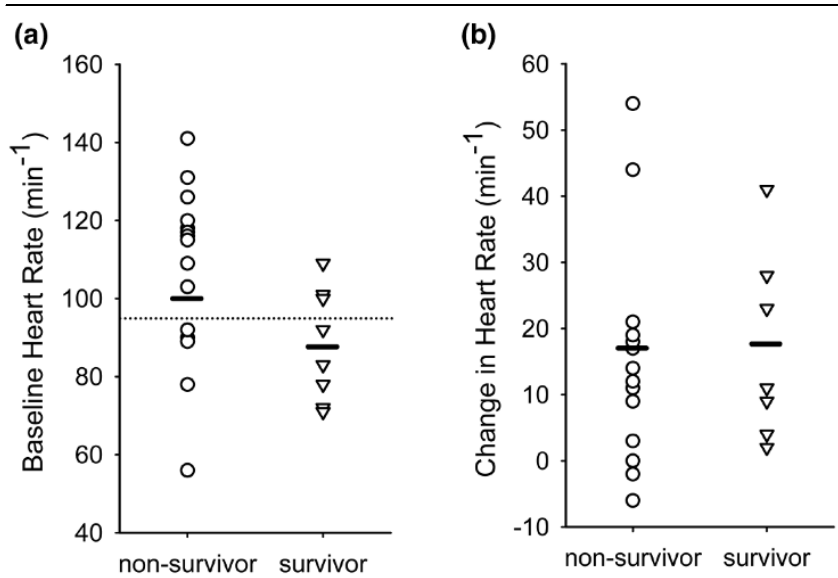

(a) Baseline heart rate in survivors and non-survivors. Dotted line indicates optimally predictive cut-off value of $95 \mathrm{beats} / \mathrm{min}$ to differentiate between survivors and non-survivors. Outcome stratification was deficient in only 8 of the 23 total subjects using this cut-off. Heart rate was the only baseline haemodynamic variable associated with outcome $(p=$ 0.0254). (b) Change in heart rate from baseline at the point of maximal cardiac output response during dobutamine challenge. Change in heart rate was not significantly different between survivors and non-survivors. Dash indicates mean value for group.
Both were non-survivors. A cut-off value of $8.5 \mathrm{~mL} / \mathrm{m}^{2}$ increase in SVI could differentiate between survivors and non-survivors in 21 of 23 cases with just one subject mismatched in each group (Figure 2b). Baseline SVI did not differentiate between survivors and non-survivors (Table 2, Figure 2a).

Other cardiac performance variables related to SVI also were associated with survival on univariate analysis including $\mathrm{Cl}$ and pulse pressure (PP)(Table 2). Greater increases in $\mathrm{DO}_{2} \mathrm{l}$, $\mathrm{MVO}_{2}$ and $\mathrm{CvO}_{2}$ and decreased OER (variables associated with $\mathrm{Cl}$ ) were similarly associated with improved outcome. However, calculated oxygen consumption as reflected by $\mathrm{VO}_{2} \mathrm{I}$ was not. Several variables associated with vascular resistance were also found to be significantly associated with survival on univariate analysis including decreases in SVRI,

\section{Figure 2}

(a)

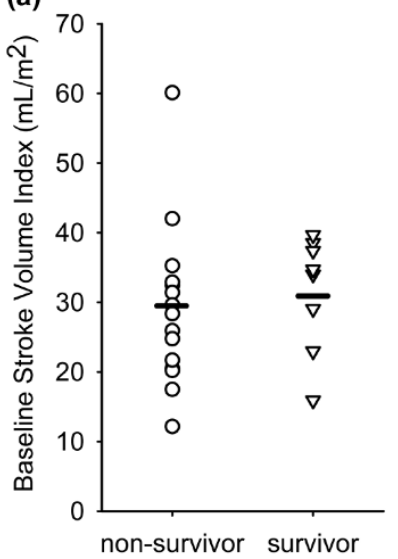

(b)

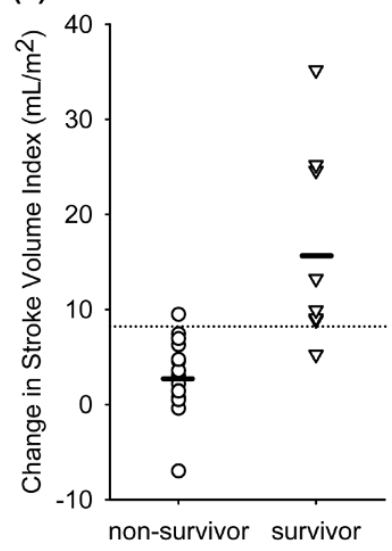

(a) Baseline stroke volume index in survivors and non-survivors. Stroke volume index at baseline was not significantly different between survivors and non-survivors. (b) Change in stroke volume index from baseline at the point of maximal cardiac output response during dobutamine challenge. Change in stroke volume was the strongest predictor of outcome among tested variables $(p=0.0003)$. A cut-off value of $8.5 \mathrm{~mL} /$ $\mathrm{m}^{2}$ increase in stroke volume index (indicated by the dotted line) correctly categorised outcome in 21 of 23 subjects with only one mismatch in each group. Dash indicates mean values. 
Figure 3

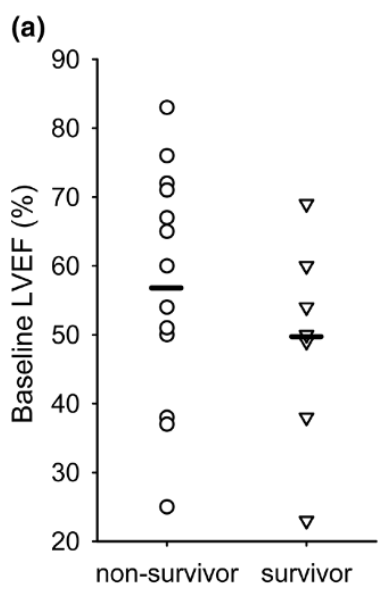

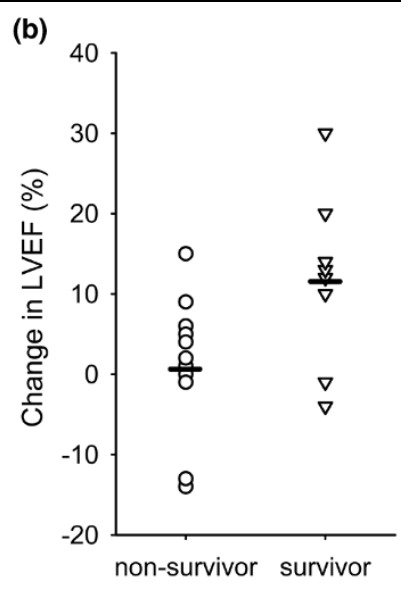

(a) Baseline left ventricular ejection fraction in survivors and non-survivors. Seven of fourteen non-survivors and five of eight survivors had a reduced ejection fraction (less than 55 per cent). The lower mean ejection fraction in survivors did not reach statistical significance in comparison to the higher ejection fraction in non-survivors. (b) Change in left ventricular ejection fraction from baseline at the point of maximum cardiac index response. The mean increase in left ventricular ejection fraction was significantly greater in survivors than in non-survivors $(p=$ 0.0160). Dash indicates mean values.

PVRI and dPAP. Decreases in diastolic systemic arterial pressure did not reach significance in this regard.

In terms of the mechanics of cardiovascular performance, greater increases of left ventricular contractility indices were associated with survival in patients with septic shock (Table 2). Indices of relevance included LVEF (Figure 3), LVSWI and Figure 4
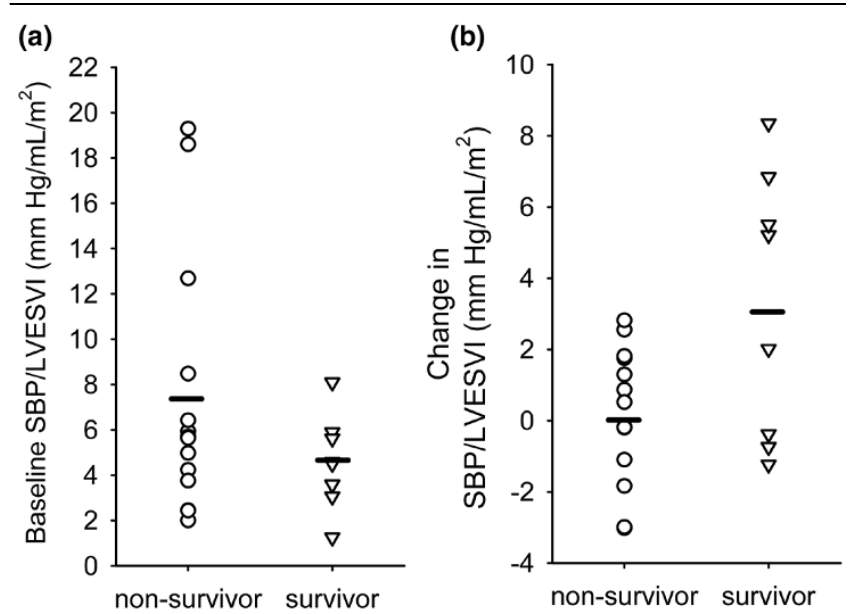

(a) Baseline peak systolic blood pressure/left ventricular end-systolic volume index (sBP/LVESVI) in survivors and non-survivors. Baseline sBP/LVESVI was not significantly different between survivors and nonsurvivors. (b) Change in SBP/LVESVI from baseline at the point of maximum cardiac output response. A significantly greater mean increase in $s B P / L V E S V I$ was seen in survivors $(p=0.0188)$. Non-survivors had almost no mean change in this parameter with dobutamine infusion. Dash indicates mean values.
Figure 5
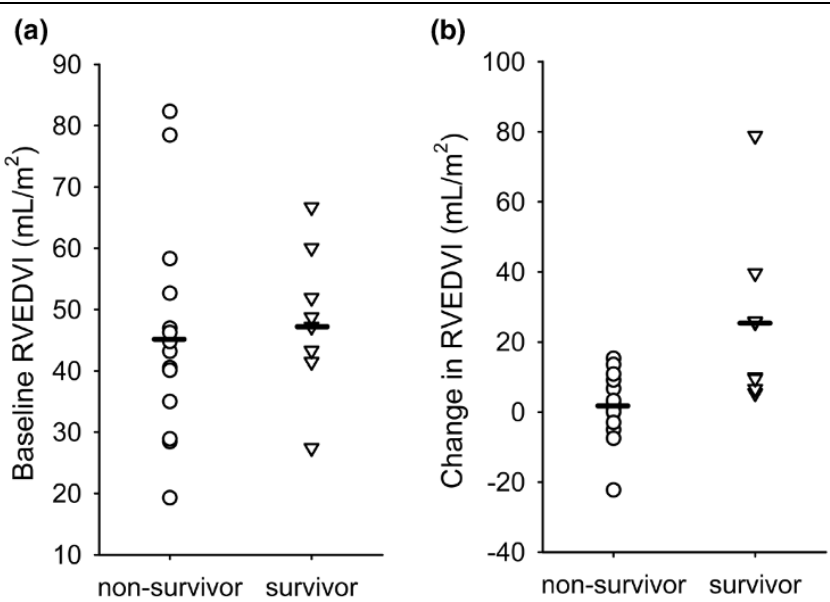

(a) Baseline right ventricular end-diastolic volume index (RVEDVI) in survivors and non-survivors. Baseline RVEDVI was similar in both survivors and non-survivors. (b) Change in RVEDVI from baseline at the point of maximum cardiac index response. The mean increase in RVEDVI was significantly greater in survivors than non-survivors $(p=$ 0.0047). Dash indicates mean values.

parameters were not similarly associated with survival. However, increases in RVEDVI (Figure 5) and right ventricular compliance (RVEDVI/RAP) either achieved or trended strongly towards significance in association to survival (Table 2).

Using multivariate forward entry discriminant analysis, several alternative models with similar predictive accuracy were identified depending on the significance level used to add and retain variables in each model. In each case, the SVI response was, by far, the most predictive variable. However, the most parsimonious discriminant model achieved maximal accuracy with the addition of sBP/LVESVI response as a 2nd factor in the model. Using this model, outcome in 22 of 23 cases could be appropriately categorised (Table 3 ). SVI responses were 43 per cent more predictive of outcome than SBP/LVESVI responses in this model (ratio of structure coefficients). Other variables associated with left ventricular contractility and right ventricular lusitropy did not contribute to prediction of outcome in the multivariate model. Multiple logistic model parameters are summarised in Table 3. Positive B coefficients in the logistic regression model indicate that higher SVI and SBP/ LVESVI are independently indicative of increased probability of survival. Confidence intervals indicate as much as a doubling of survival odds, on average, over the range of SVI and sBP/LVESVI responses covered by this sample [14]. Discriminant multivariate assessment of relative (indexed to baseline) changes in responses yielded similar results.

\section{Discussion}

Dobutamine, a racemic synthetic catecholamine composed of two enantiomers with divergent sympathetic activity, was developed as an inotropic agent and is most commonly used for support of patients with congestive heart failure and other 
low output states of cardiogenic decompensation [17]. A series of studies have assessed this compound as a potential adjunctive therapy in severe sepsis for purposes of augmentation of oxygen delivery [18-21]. In addition, a dobutamine stress test with determination of oxygen consumption responses to assess survival potential of patients with severe sepsis and septic shock has been examined [22-24]. No studies have assessed volumetric responses to graded dobutamine infusion in septic patients in relationship to outcome.

This study, like that of Jellema and colleagues [25], shows that dobutamine infusion in patients with septic shock generates a highly heterogenous cardiovascular response. We also demonstrate that dobutamine-stressed ventricular and cardiovascular responses are highly correlated with outcome. The observation that greater increases in left ventricular contractility (LVEF, sBP/LVESVI, LVSWI) and right ventricular lusitropy (RVEDVI, RVEDVI/RAP) indices are associated with survival in severe sepsis and septic shock in univariate analysis, is entirely novel. In addition, an independent contribution to prediction of survival under dobutamine stress from cardiovascular performance (SVI) and left ventricular contractility (sBP/LVESVI) responses has also not been previously reported. Notably, the correlative strength of these responses is substantially more powerful than that generated by initial $\mathrm{HR}$, the only baseline hemodynamic index to predict outcome.

These results are important for at least three reasons. First, they support the notion that simple haemodynamic responses to dobutamine stress can potentially differentiate between survivors and non-survivors of sepsis and septic shock. This confirms long-standing clinical observations and suggests that these responses may be useful in terms of clinical prognostication for purposes of risk stratification in clinical trials. Second, they suggest that dynamic cardiovascular responses may be superior to static performance parameters with respect to their association with survival. Finally, these observations provide important insights regarding the mechanism underlying the cardiovascular response to adrenergic stimulation in survivors versus non-survivors of septic shock.

The observation that only HR among all baselines haemodynamic/oxygen metabolism variables assessed was significantly correlated with outcome has precedence in other studies of septic shock $[4,26]$. Azimi and coworkers demonstrated that only heart rate among initial haemodynamic variables was associated with outcome with a value of less than 106 beats/min linked to survival [26]. No other initial parameters were associated with prognosis. Parker and colleagues similarly demonstrated that HR of less than 95 beats/min at presentation was associated with survival in a study of 48 patients with septic shock [4]. There are several possibilities to explain the association of an increased HR with a higher probability of death. The increased HR in non-survivors may indicate a higher level of sepsis-induced cardiovascular stress due to a more severe disease state. Alternately, it may represent a secondary compensatory response as a consequence of decreased sensitivity to the direct myocardial inotropic effects of endogenous catecholamines in patient with particularly severe septic myocardial dysfunction $[3,27]$. Given that baseline SV and SBPVESI was similar in both groups, this possibility seems less likely. Another possibility is that non-survivors have increased sensitivity to catecholamine stimulation. This also seems unlikely since several studies have shown $\beta$ adrenergic uncoupling in myocardial tissue exposed to inflammatory mediators such as those found circulating in patients with septic shock $[28,29]$. Since the study was not designed to determine the cause of this finding, no definitive conclusion can be drawn.

Despite observations dating back several decades suggesting that reduced response to catecholamine support in septic shock is highly predictive of poor outcome, most recent work examining the prognostic significance of dobutamine stress in this situation has focused on oxygen consumption/delivery indices [22-24]. Both Vallet et al [24] and Rhodes et al [22] have shown that increases of oxygen consumption of greater than 15 per cent with $10 \mu \mathrm{g} / \mathrm{kg} / \mathrm{min}$ during severe sepsis are associated with a favorable outcome. However, it is not at all clear that it is necessary to invoke oxygen consumption/delivery responses for predictive power. Both studies clearly demonstrated that cardiac index responses were also highly associated with outcome. However, neither closely examined cardiac performance parameters as correlates of outcome.

In our study, cardiovascular performance as indicated by SVI response was the dominant correlate to outcome with a single cut off value of $8.5 \mathrm{~mL} / \mathrm{min}$ able to differentiate 21 of 23 outcomes. The predictive power of most other parameters appeared to be physiologically linked to this response and did not persist in multivariate analysis (Cl, PP, OER, PAD, SVRI, PVRI, $\mathrm{DO}_{2} \mathrm{I}, \mathrm{MvO}_{2}, \mathrm{CvO}_{2}$ ). sBP/LVESVI, a relatively load-independent index of left ventricular contractility [30], was the only variable that possessed predictive power independent of stroke volume index (in analysis with both absolute and relative changes from baseline). This response appears to statistically dominate other contractility indices (LVEF and LVSWI) in terms of contribution to the model. If SBP/LVESVI is removed from the model, then LVEF becomes a significant contributor along with SVI.

The fact that both cardiac performance and contractility increases in response to dobutamine stress are highly associated with outcome in septic shock is consistent with the possibility that the presence of cardiovascular reserve (that is an indication of submaximal cardiovascular response to baseline stress) may be critical to survival. This concept of preserved physiologic reserve as a marker of good prognosis in septic shock has previously been postulated by Hayes et al [31] and Rhodes et al [22] albeit in the context of oxygen consumption 
indices. However, it may relate directly to cardiovascular performance and contractility indices as shown in this study.

This study may also demonstrate that the concept of preload reserve used most commonly in reference to ischemic heart disease associated limitations in cardiac performance may also apply to septic shock [32]. Several groups of investigators have shown that subjects with clinically asymptomatic impairment of systolic cardiac function can be differentiated from symptomatic patients by the presence of exercise stressinduced increases in SVI in association with ventricular dilatation $[33,34]$. No direct link has yet been made between survival and the presence of this phenomenon among patients with impaired heart function. Despite this, our study may be unmasking a parallel, related phenomenon in that both exercise and dobutamine challenge represent stressed states in comparison to baseline.

Similarly, our observations of an association of right ventricular dilatation (Table 2, Figure 5a) in response to dobutamine (with a similar trend for the left ventricle) appear to be related to our previous observations which demonstrate a relationship between right and left ventricular dilation and survival in septic shock $[3,8,35]$.

One possibility to tie these observations together is that recruitment of preload reserve is a critical but late response during high degrees of cardiovascular stress. If that is the case, then a lack of ability to recruit ventricular volume at any point during cardiovascular stress may indicate that the subject is already operating at the maximum efficacy of the FrankStarling response and has no additional cardiovascular reserve. In this formulation, patients with chronic systolic dysfunction would be more likely to be symptomatic, while patients with septic shock would be more likely to die if such reserve was limited. The nature of the limitation of cardiovascular reserve in conditions of high stress would be a combination of the baseline cardiovascular fitness and the degree of acute cardiovascular stress.

The study demonstrates that cardiovascular hypo-responsiveness to catecholamine stimulation is strongly associated with mortality in severe sepsis and septic shock. Compared to baseline differences in heart rate, dobutamine stress-induced variations in stroke volume responses are much more closely related to outcome. Dobutamine-induced differences in contractility responses also appear to possess independent correlative power with respect to mortality. One physiological mechanism supporting increased cardiac output appears to involve ventricular dilatation in survivors suggesting an important role for "preload reserve" in optimal cardiovascular response to severe sepsis and septic shock. This response may be related to previous observations demonstrating that increased ventricular dilatation is characteristic of survival from septic shock.
This study makes several novel observations. However there are significant limitations to the results. First, despite the fact that dobutamine-stress induced cardiovascular responses are highly associated with outcome, their utility for risk stratification as related to research or clinical prognostication remains unproven. A prospective study would be required to validate their utility for that purpose. Second, these data do not suggest that any of the variables associated with outcome drive these are responsible for these outcomes. It is much more likely that these associations are correlations without causality. For example, reduced stroke volume response to dobutamine likely indicates underlying severity of illness rather than being a direct contributor to risk of death. Finally, there may be significant unmeasured confounders for some of the documented responses. Higher mean intra-thoracic pressures due to more severe lung disease and greater ventilatory requirements could contribute to the smaller RVEDVI and RVEDVI/RAP responses to dobutamine. These patients could also be at higher risk of death. Unfortunately, mean airway pressures are not available for analysis.

\section{Conclusion}

Although a variety of dobutamine-stimulated cardiovascular and ventricular responses are associated with outcome in sepsis and septic shock, SVI increases are most strongly correlated with survival. Since this stimulation test involves transient administration of dobutamine, this response is probably indicative of preservation of cardiac signalling mechanisms and of cardiovascular reserve in the septic patient. Notably, the predictive strength of these responses is substantially more powerful than that generated by initial $\mathrm{HR}$, the only baseline haemodynamic index associated with outcome.

Further research is required to better understand the mechanisms underlying differing cardiovascular responses during severe sepsis and septic shock both at baseline and under exogenous catecholamine stress.

\section{Key Messages}

1) Survival from sepsis and septic shock is closely associated with dobutamine-stimulated increases in stroke volume.

2) Survivors of sepsis and septic shock are characterised by preserved cardiovascular reserve including preload reserve.

\section{Competing interests}

The authors declare that they have no competing interests.

\section{Authors' contributions}

Anand Kumar had overall responsibility for this study including study design, administration, and execution. He was also was primarily responsibility for data analysis and manuscript development. Elizabeth Schupp (study execution), Eugene Bunnell (study execution), Amjad Ali (nuclear ventriculography) and Barry Milcarek (statistics) assisted with specific aspects of the 
project. Joseph Parrillo was involved in study design and manuscript preparation.

\section{References}

1. Weil $\mathrm{MH}$, Nishijima $\mathrm{H}$ : Cardiac output in bacterial shock. $A m \mathrm{~J}$ Med 1978, 64:920-922.

2. Wilson RF, Thal AP, Kindling PH, Grifka T, Ackerman E: Hemodynamic measurements in septic shock. Arch Surg 1965, 91:121-129.

3. Parker MM, Shelhamer JH, Bacharach SL, Green MV, Natanson C, Frederick TM, Damske BA, Parrillo JE: Profound but reversible myocardial depression in patients with septic shock. Ann Intern Med 1984, 100:483-490.

4. Parker MM, Shelhamer JH, Natanson C, Alling DW, Parrillo JE: Serial cardiovascular variables in survivors and nonsurvivors of human septic shock: Heart rate as an early predictor of prognosis. Crit Care Med 1987, 15:923-929.

5. MacLean LD, Mulligan WG, McLean APH, Duff JH: Patterns of septic shock in man: A detailed study of 56 patients. Ann Surg 1967, 166:543-562.

6. Groenveld ABJ, Nauta JJ, Thijs L: Peripheral vascular resistance in septic shock: its relation to outcome. Intensive Care Med 1988, 14:141-147.

7. Tuchschmidt J, Fried J, Astiz M, Rackow EC: Elevation of cardiac output and oxygen delivery improves outcome in septic shock. Chest 1992, 102:216-220.

8. Parker MM, McCarthy KE, Ognibene FP, Parrillo JE: Right ventricular dysfunction and dilatation, similar to left ventricular changes, characterize the cardiac depression of septic shock in humans. Chest 1990, 97:126-131.

9. Kimchi A, Ellrodt GA, Berman DS, et al:: Right ventricular performance in septic shock: a combined radionuclide and hemodynamic study. JACC 1984, 4:945-951.

10. Ellrodt AG, Riedinger MS, Kimchi $A$, et al.: Left ventricular performance in septic shock: Reversible segmental and global abnormalities. Am Heart J 1985, 110:402-409.

11. Ognibene FP, Parker MM, Natanson $\mathrm{C}$, Shelhamer JH, Parrillo JE: Depressed left ventricular performance. Response to volume infusion in patients with sepsis and septic shock. Chest 1988, 93:903-910.

12. Weisel RD, Vito L, Dennis RC, Hechtman HB: Myocardial depression during sepsis. Am J Surg 1977, 133:512-521.

13. Levy B, Dusang B, Annane D, Gibot S, Bollaert PE, College Interregional des Réanimateurs du Nord-Est: Cardiovascular response to dopamine and early prediction of outcome in septic shock: a prospective multiple-center study. Crit Care Med 2005, 33(10):2172-2177.

14. Bone RC, Balk RA, Cerra FB, Dellinger RP, Fein AM, Knaus WA, Schein RM, Sibbald WJ: ACCP/SCCM Consensus Conference: Definitions for sepsis and organ failure and guidelines for use of innovative therapies in sepsis. Chest 1992,101:1644-1655.

15. Morrison DA, Turgeon J, Ovitt T: Right ventricular ejection fraction measurement: Contrast ventriculography versus gated blood pool and gated first-pass radionuclide methods. $A m \mathrm{~J}$ Cardiol 1984, 54:651-653.

16. Port SC: Recent advances in first-pass radionuclide angiography. Cardiology Clinics of North America 1994, 12:359-372.

17. Leier CV, Unverferth DV: Dobutamine. Ann Intern Med 1983, 99:490-496.

18. Shoemaker WC, Appel PL, Kram HB: Hemodynamic and oxygen transport effects of dobutamine in critically ill general surgical patients. Crit Care Med 1986, 14:1032-1037.

19. Vincent JL, Roman A, De Backer D, Kahn RJ: Oxygen uptake/ supply dependency: Effects of short-term dobutamine infusion. Am Rev Respir Dis 1990, 142:2-8.

20. Vincent JL, Roman A, Kahn RJ: Dobutamine administration in septic shock: addition to a standard protocol. Crit Care Med 1990, 18:689-693.

21. Hayes MA, Timmins AC, Yau EH, Palazzo M, Watson D, Hinds CJ: Oxygen transport patterns in patients with sepsis syndrome or septic shock: influence of treatment and relationship to outcome. Crit Care Med 1997, 25(6):926-936.

22. Rhodes A, Lamb FJ, Malagon I, Newman PJ, Grounds RM, Bennett ED: A prospective study of the use of a dobutamine stress test to identify outcome in patients with sepsis, severe sepsis or septic shock. Crit Care Med 1999, 27(11):2361-2366.

23. De Backer D, Moraine JJ, Berre J, Kahn RJ, Vincent JL: Effects of dobutamine on oxygen consumption in septic patients. Direct versus indirect determinations. American Journal of Respiratory and Crit Care Med 1994, 150(1):95-100.

24. Vallet B, Chopin C, Curtis SE: Prognostic value of the dobutamine test in patients with sepsis syndrome and normal lactate values: A prospective, multicenter study. Crit Care Med 1993, 21:1868-1875

25. Jellema WT, Groeneveld AB, Wesseling KH, Thijs LG, Westerhof $\mathrm{N}$, van Lieshout JJ: Heterogeneity and prediction of hemodynamic responses to dobutamine in patients with septic shock. Crit Care Med 2006, 34(9):2392-2398.

26. Azimi G, Vincent JL: Ultimate survival from septic shock. Resuscitation 1986, 14:245-253.

27. Parrillo JE, Burch $\mathrm{C}$, Shelhamer JH, Parker MM, Natanson C, Schuette W: A circulating myocardial depressant substance in humans with septic shock. Septic shock patients with a reduced ejection fraction have a circulating factor that depresses in vitro myocardial cell performance. J Clin Invest 1985, 76:1539-1553

28. Kumar A, Paladugu B, Mensing J, Kumar A, Parrillo JE: Nitric oxide-dependent and - independent mechanisms are involved in TNF-alpha-induced depression of cardiac myocyte contractility. Am J Physiol - Regulatory Integrative \& Comparative Physiology 2007, 292(5):R1900-R1906.

29. Silverman HJ, Penaranda R, Orens JB, Lee NH: Impaired $\beta$ adrenergic receptor stimulation of cyclic adenosine monophosphate in human septic shock: Association with myocardial hyporesponsiveness to catecholamines. Crit Care Med 1993, 21:31-39.

30. Carabello BA, Spann JF: The uses and limitations of end-systolic indices of left ventricular function. Circulation 1984, 69:1058-1064.

31. Hayes MA, Timmins AC, Yau EH, Palazzo M, Hinds CJ, Watson D: Elevation of systemic oxygen delivery in the treatment of critically ill patients. N Engl J Med 1994, 330:1717-1722.

32. Ross J Jr, Ross JJ: Afterload mismatch and preload reserve: a conceptual framework for the analysis of ventricular function. [Review] [70 refs]. Progress in Cardiovascular Diseases 1976, 18(4):255-264.

33. Konstam MA, Kronenberg MW, Udelson JE, Kinan D, Metherall J, Dolan N, Edens T, Howe D, Kilcoyne L, Benedict C, et al.: Effectiveness of preload reserve as a determinant of clinical status in patients with left ventricular systolic dysfunction. The SOLVD Investigators. Am J Cardiol 1992, 69(19):1591-1595.

34. Dahan M, Aubry N, Baleynaud S, Ferreira B, Yu J, Gourgon R: Influence of preload reserve on stroke volume response to exercise in patients with left ventricular systolic dysfunction: a Doppler echocardiographic study. JACC 1995, 25(3):680-686.

35. Parrillo JE, Parker MM, Natanson C, Suffredini AF, Danner RL, Cunnion RE, Ognibene FP: Septic shock in humans. Advances in the understanding of pathogenesis, cardiovascular dysfunction, and therapy. Ann Intern Med 1990, 113:227-242. 